DOI: $10.12731 / 2070-7568-2020-1-173-183$

УДК 004.9

\title{
МОБИЛЬНЫЕ ПРИЛОЖЕНИЯ \\ КАК ИНСТРУМЕНТ ОРГАНИЗАЦИИ НЕТВОРКИНГА
}

\section{Сухоруков Н.Н.}

Нетворкинг - это относительно новое понятие. Тем не менее, ежегодно только в России проводится несколько сотен нетворкинг-мероприятий. Нельзя недооченивать важность нетворкинга - ведь именно он позволяет решать самый широкий круг задач в личной жизни и бизнесе. Нетворкинг является одним из самых эффективных и наименее дорогих инструментов расширения соииального капитала и ведения бизнеса. Однако, организачия и проведение нетворкинг-мероприятия-это долгий, трудоемкий проиесс и уже сейчас эта деятельность может быть частично автоматизирована с помощью мобильных приложений. Статья содержит исследование перспектив применения мобильных приложений для организации и проведения нетворкинга, а также повымения эффективности коммуникации на мероприятиях подобного уровня. В данной статье рассмотрена сфера организации нетворкинг-мероприятий, определены ее главные проблемы и пути их решения. Также рассмотрен ряд популярных приложений для организачии нетворкинга, выявлены их преимущества и недостатки, даны рекомендации по улучшению их функциональных возможностей.

Цель работы: определение проблем сферы организации нетворкинг-мероприятий, идентификация факторов, влияющих на эффективность коммуникации, а также анализ перспектив использования специализированных мобильных приложений для организации нетворкинга.

Метод или методология проведения работы: в статье использовались эмпирические методы (сравнение и анкетирование), методы теоритического исследования (анализ и синтез). 
Результаты: сформулированы проблемы сферы организации нетворкинг-мероприятий и предложень пути их решения, разработаны рекомендации по совершенствованию функциональных возможностей мобильных приложений для организации нетворкинга, а также разработаны ключевые показатели эффективности (КРI) для оценки эффективности коммуникации.

Область применения результатов. Результаты анализа могут быть использованы разработчиками прикладного программного обеспечения, а также профессиональньми организаторами нетворкинг-мероприятий.

Новизна исследования. Состоит в разработке рекомендаций по улучшению функииональных возможностей мобильных приложений для организации нетворкинга, а также в разработке ключевых показателей эффективности для оценки эффективности коммуникации.

Ключевые слова: нетворкинг; организация мероприятия; приложения для нетворкинга; phydgital.

\section{MOBILE APPLICATIONS \\ AS A TOOL FOR ORGANISING NETWORKING}

\section{Sukhorukov N.N.}

Networking - it is a relatively new concept. Nevertheless, several hundred networking-events are held annually in Russia alone. The importance of networking cannot be underestimated - it allows us to solve a wide range of tasks in personal life and business. Networking is one of the most effective and least expensive tools for expanding social capital and doing business. However, the organization and conduct of networking events is a long, laborious process but now this activity can be partially automated by using mobile applications.

The article contains a study of the prospects of using mobile applications for organizing and conducting networking, as well as improving the effectiveness of communication at events of this level. This article 
discusses the scope of organization of networking events, identifies its main problems and the ways to solve them. A number of popular applications for organizing networking are also considered, their advantages and disadvantages are identified, and recommendations for improving their functionality are given.

Purpose: to define the problems in the sphere of organizing networking events, the identification of factors that affect the effectiveness of communication, as well as analysis of prospects for using specialized mobile applications for networking.

Method or methodology of the work: the article used empirical methods (comparison and survey), and methods of theoretical research (analysis and synthesis).

Results: the problems of the organization of networking events are formulated and the ways to solve them are proposed, the recommendations are developed for improving the functionality of mobile applications for networking, and the key performance indicators (KPIS) are developed to evaluate communication efficiency.

Practical implications. Developers of application software, as well as professional organizers (moderators) of networking events can use the results of the analysis.

The novelty of the study. Consist in developing recommendations for improving the functionality of mobile applications for networking, as well as developing key performance indicators to assess the effectiveness of a communication.

Keywords: networking; event organization; networking apps; phydgital.

\section{Введение}

Проведение управляемых (фасилитируемых) деловых совещаний, конференций, коллективных дискуссий и нетворкинг-мероприятий является новым, восходящим трендом. Под нетворкингом принято понимать такую деятельность, целью которой является «быстрое и успешное решение задач самых разных уровней сложности» [5] как в личной жизни, так и в бизнесе с помощью расши- 
рения круга знакомств и создания сети полезных контактов. Нетворкинг является «одной из современных, конструктивных идей», реализация которой помогает предпринимателям добиться успеха [1] и все больше людей для поиска новых деловых возможностей, единомышленников, компаний и новых источников профессиональных знаний предпочитают использовать нетворкинг [14].

Однако, организация подобных мероприятий имеет ряд проблем и ограничений. К таковым относят: а) высокую стоимость организации мероприятия; б) субъективное влияние модератора на процесс дискуссии; в) сложность управления большой группой участников мероприятия; г) нарушение режима времени и регламента мероприятия; д) большое время сбора и обработки обратной связи от участников.

\section{Актуальность}

Решение указанных выше проблем позволит повысить результативность подобных мероприятий, а именно позволит создать новые связи между представителями бизнеса, соединить людей с ресурсами, упростить обмен ресурсами и идеями и ускорить инновационный процесс.

В настоящее время отрасль деловых мероприятий «подвергается трансформации, благодаря новым возможностям, которые несут в себе информационные технологии» [4, с. 44-48]. Уже сейчас существуют приложения и веб-сервисы, призванные устранить некоторые проблемы офлайн нетворкинга и обеспечить его цифровую поддержку, тем самым создав новую реальность - «Phydgital» (от англ. physical + digital - физический и цифровой), т.е. создать коммуникацию на стыке двух пространств - физического и цифрового.

\section{Материалы и методы исследования}

Для проведения исследования были отобраны 6 приложений и веб-сервисов для организации нетворкинга и сбора обратной связи от участников: Eventsxd [8], Meyou [10], LineUpr [9], Worksup [15], Whova [6] и Bizzabo [7]. Данные приложения были детально 
изучены (таблица 1), после чего был составлен список полезных существующих функций и список недостающих, требующих реализации функций.

\section{Результаты исследования}

Организаторы мероприятий все чаще для своей работы используют социальные сети и специализированные приложения для работы со своей аудиторией [3, с. 470-471]. Ряд авторов указывает на рост числа приложений для нетворкинга, возрастающий к ним интерес [2] и выявляют их некоторые проблемы [4, с. 44-48]. Другие исследователи подчеркивают исключительную роль нетворкинга для развития бизнеса $[13$, с. 176] и важность использования нетворкинг-приложений [12, с. 20].

Современный рынок мобильный и веб-приложений для организации и проведения мероприятий предлагает большой выбор различных решений. Условно их можно подразделить на две группы: а) приложения для работы с аудиторией, показа презентаций и сбора обратной связи в реальном времени; б) приложения для знакомства и коммуникации участников на мероприятии. В данной статье рассматривается именно вторая группа приложений.

Таблица 1.

Сравнительная характеристика приложений для нетворкинга

\begin{tabular}{|c|c|c|c|c|c|c|c|}
\hline & \multirow{2}{*}{ Критерии } & \multicolumn{6}{|c|}{ Анализируемые приложения и веб-сервисы } \\
\hline & & Eventsxd & MeYou & LineUpr & Worksup & Whova & Bizzabo \\
\hline \multirow{5}{*}{ 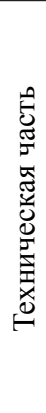 } & $\begin{array}{l}\text { Кроссплатформен- } \\
\text { ность }\end{array}$ & Да & Да & Да & Да & Да & Да \\
\hline & $\begin{array}{l}\text { Интуитивно понят- } \\
\text { ный интерфейс }\end{array}$ & Нет & Да & Да & Да & Нет & Да \\
\hline & $\begin{array}{l}\text { Авторизация через } \\
\text { сторонние сервисы }\end{array}$ & Нет & Да & Да & Нет & Да & Нет \\
\hline & $\begin{array}{l}\text { Поддержка мульти- } \\
\text { зальности / мульти- } \\
\text { экранности } \\
\end{array}$ & Нет & Да & Нет & Да & Нет & Нет \\
\hline & $\begin{array}{l}\text { Поддержка мульти- } \\
\text { язычности }\end{array}$ & Нет & Да & Да & Нет & Нет & Да \\
\hline
\end{tabular}


Окончание табл. 1.

\begin{tabular}{|c|c|c|c|c|c|c|c|}
\hline \multirow{6}{*}{ 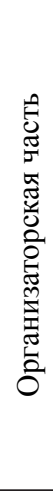 } & $\begin{array}{l}\text { Возможность соз- } \\
\text { дания опросов }\end{array}$ & Да & Да & Нет & Да & Да & Нет \\
\hline & $\begin{array}{l}\text { Возможность соз- } \\
\text { дания анкет }\end{array}$ & Да & Нет & Нет & Да & Да & Нет \\
\hline & $\begin{array}{l}\text { Выгрузка аналити- } \\
\text { ки в excel/pdf }\end{array}$ & Да & Да & Да & Да & & Да \\
\hline & $\begin{array}{l}\text { Наличие системы } \\
\text { поощрения участ- } \\
\text { ников }\end{array}$ & Нет & Да & Нет & Нет & Нет & Нет \\
\hline & $\begin{array}{l}\text { Наличие профиля } \\
\text { пользователя }\end{array}$ & Да & Да & Да & Да & Да & Да \\
\hline & $\begin{array}{l}\text { Возможность брен- } \\
\text { дировать интерфейс }\end{array}$ & Да & Да & Нет & Нет & Нет & Да \\
\hline \multirow{6}{*}{ 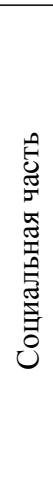 } & $\begin{array}{l}\text { Просмотр списка } \\
\text { участников }\end{array}$ & Да & Да & Нет & Да & Да & Да \\
\hline & $\begin{array}{l}\text { Наличие кнопки } \\
\text { «Поделиться в соц. } \\
\text { сетях» }\end{array}$ & Нет & Нет & Да & Нет & Нет & Нет \\
\hline & $\begin{array}{l}\text { Чат с организато- } \\
\text { рами }\end{array}$ & Нет & Да & Нет & Да & Да & \\
\hline & Чат с участниками & Да & Да & Нет & Да & Да & Да \\
\hline & $\begin{array}{l}\text { Функционал для } \\
\text { знакомства гостей }\end{array}$ & Нет & Да & Нет & Нет & Нет & Нет \\
\hline & $\begin{array}{l}\text { Сопоставление } \\
\text { участников по ин- } \\
\text { тересам }\end{array}$ & Нет & Нет & Нет & Да & Нет & Нет \\
\hline
\end{tabular}

По результатам проведенного анализа были выявлены удачные решения, такие как: реализация кроссплатформенности, авторизации через социальные сети, поддержки нескольких языков, возможности создания опросов, анкет (в том числе содержащих открытые вопросы), инструментов вовлечения участников в нетворкинг и их поощрения, инструментов просмотра аналитики и выгрузки отчетов мероприятия, профиля пользователя с подробной информацией о нем, чата с организатором мероприятия и программы мероприятия.

Недостающим у подавляющего большинства приложений, но крайне важными элементом является инструмент для знакомства гостей мероприятия. Эти же выводы были подтверждены в результате опроса участников (организаторов и гостей) нетворкинг-мероприятий. 
Проблема любого офлайн нетворкинга заключается в том, что участник мероприятия должен сам проявлять инициативу и взаимодействовать с другими участниками. У некоторых из них могут быть внутренние барьеры, мешающие активно знакомиться и взаимодействовать с другими, либо наоборот участник может потратить большую часть времени на общение с одним или несколькими участниками. Поэтому необходима реализация такого функционала, который будет принудительно «сталкивать» участников друг с другом, ограничивать время на общение и позволит каждому участнику мероприятия установить контакт с максимально возможным количеством.

Для отслеживания эффективности коммуникации возможно использование следующих показателей (KPI):

1) Общее количество заключенных договоренностей во время мероприятия;

2) Количество заключенных договоренностей конкретным участником;

3) Количество получаемых ресурсов некоторого типа;

4) Количество отдаваемых ресурсов некоторого типа;

5) Количество запрашиваемых ресурсов некоторого типа;

6) Количество уникальных контактов одного участника с другими;

7) Время взаимодействия участников друг с другом.

Таким образом, эффективность коммуникации достигается за счет: а) достижения целей, поставленных модератором перед участниками; б) соблюдения регламента дискуссии, временных рамок и обсуждением только заданной темы; в) вовлечения всех участников в процесс дискуссии; г) наличия обратной связи от участников (опросы, анкеты, голосования) и ее визуализации.

Исходя из вышесказанного, становится очевидным, что приложения для нетворкинга должны быть ориентированы и адаптированы к созданию и поддержанию отношений между людьми [12], что облегчит приобретение знаний, навыков и ресурсов, необходимых для роста и выживания как самого человека, так и его бизнеса [13]. 


\section{Заключение}

Любое приложение, предназначенное для организации деловых мероприятий, имеет те или иные недостатки, но уже сейчас положительный социальный эффект от их использования заключается в создании четкого, управляемого процесса взаимодействия людей, формировании нормы дискуссии и создании условий, способствующих передаче знаний, и ускорению инновационного процесса [11]. Интеграция онлайн и офлайн взаимодействия происходит во многих областях человеческой деятельности, что обеспечивает «повышение эффективности производства, организуя новые экономические, политические и общественные структуры» [2], что позволяет ускорить процесс обмена идеями и ресурсами, что в конечном счете будет способствовать развитию предпринимательства и цифровой экономики в целом.

Таким образом, проведенное исследование позволило выявить ряд проблем, присущих сфере организации нетворкинга. Рассмотренные приложения для нетворкинга позволяют частично или полностью решить выявленные проблемы, а введение метрик оценки эффективности коммуникации позволит оценить результативность каждого отдельно взятого нетворкинг-мероприятия, выделить лучшие практики или провести работу над ошибками.

\section{Список литературы}

1. Ефимова И.Е., Крутиков В.К., Цоп-Петрова И. Новый алгоритм поведения людей: нетворкинг // Вестник образовательного консорциума среднерусский университет. Серия: экономика и управление. 2015. №5. C. 91-93.

2. Кушков Е.А. Популяризация мессенджеров и социальных сетей в обществе и их влияние на экономику [Электронный ресурс] // Управление экономическими системами: электронный научный журнал: [сайт] http://elib.fa.ru/art2019/bv1895.pdf(дата обращения: 02.02.2020).

3. Ли Г.С. Использование мобильных приложений для деловых МICЕмероприятий // Вестник современных исследований. 2018. №11.7. C. $470-471$. 
4. Митрейкин И.П., Шевченко Д.Е. Организация нетворкинга с помощью мобильного приложения // Молодежный научный вестник. 2016. №8. C. 44-48.

5. Столыпинская 3.И., Кокорина Е.Г. Нетворкинг: понятие, история, уровни // Сборник научных трудов научно-практической конференции для студентов и молодых ученых. Симферополь: Общество с ограниченной ответственностью «Издательство Типография «Ариал», 2017. С. 391-392.

6. Award-winning Event Apps and Event Management Software: [Электронный ресурc]: [сайт] https://www.whova.com (дата обращения: 19.01.2020).

7. Bizzabo is the world's most loved event software: [Электронный ресурс]: [сайт] https://www.bizzabo.com (дата обращения: 19.01.2020).

8. EventsXD: Mobile Conference and Event App: [Электронный ресурс]: [сайт] https://www. eventsxd.com (дата обращения: 19.01.2020).

9. LineUpr: Create Event Apps in Minutes by Yourself: [Электронный pecypc] : [сайт] https://www.lineupr.com (дата обращения: 19.01.2020).

10. MeYou - сервис для полезных деловых знакомств: [Электронный pecypc] : [сайт] https://www. meyou.ru (дата обращения: 19.01.2020).

11. Pittaway L., Robertson M., Munir K., Denyer D. and Neely A. Networking and innovation: a systematic review of the evidence // International Journal of Management Reviews, 2004, no. 5-6: pp. 137-168. DOI:10.1111/j.1460-8545.2004.00101.x

12. Rainer A., Elgar F. Business Networking Systems: Characteristics and Lessons Learned // International Journal of Electronic Commerce, 2000, no. 5:2, pp. 7-27. DOI: 10.1080/10864415.2000.11044202

13. Schoonjans B., Van Cauwenberge, P. \& Vander Bauwhede, H. Formal business networking and SME growth. Small Bus Econ, 2013, no. 41, pp. 169-181. DOI:10.1007/s11187-011-9408-6

14. Wilson J. Social networking: the business case // Engineering \& Technology, 2009, no. 4, pp. 54-56. DOI: 10.1049/et.2009.1010

15. Worksup - Interaction and Networking App for Events: [Электронный ресурс]: [сайт] https://www.worksup.com (дата обращения: 19.01.2020). 


\section{References}

1. Efimova I.E., Krutikov V.K., Tsop-Petrova I. Novyy algoritm povedeniya lyudey: netvorking [The new algorithm of human behavior: networking]. Vestnik obrazovatel'nogo konsortsiuma srednerusskiy universitet. Seriya: ekonomika i upravlenie. 2015. no. 5, pp. 91-93.

2. Kushkov E.A. Populyarizatsiya messendzherov i sotsial'nykh setey v obshchestve i ikh vliyanie na ekonomiku [Popularization of instant messengers and social networks in society and their impact on the economy]. Upravlenie ekonomicheskimi sistemami. http://elib.fa.ru/art2019/bv1895. pdf (accessed: 02.02.2020).

3. Li G.S. Ispol'zovanie mobil'nykh prilozheniy dlya delovykh MICE-meropriyatiy [Using mobile apps for business MICE events]. Vestnik sovremennykh issledovaniy. 2018. no. 11.7, pp. 470-471.

4. Mitreykin I.P., Shevchenko D.E. Organizatsiya netvorkinga s pomoshch'yu mobil'nogo prilozheniya [Organizing networking using a mobile app]. Molodezhnyy nauchny vestnik. 2016. no. 8, pp. 44-48.

5. Stolypinskaya Z.I., Kokorina E.G. Netvorking: ponyatie, istoriya, urovni [Networking: a concept, a history, a levels]. Sbornik nauchnykh trudov nauchno-prakticheskoy konferentsii dlya studentov i molodykh uchenykh [Collection of scientific papers of the scientific-practical conference for students and young scientists]. Simferopol': Obshchestvo s ogranichennoy otvetstvennost'yu «Izdatel'stvo Tipografiya «Arial», 2017, pp. 391-392.

6. Award-winning Event Apps and Event Management Software. https:// www.whova.com (accessed: 19.01.2020).

7. Bizzabo is the world's most loved event software. https://www.bizzabo. com (accessed: 19.01.2020).

8. EventsXD: Mobile Conference and Event App. https://www. eventsxd. com (accessed: 19.01.2020).

9. LineUpr: Create Event Apps in Minutes by Yourself. https://www.lineupr. com (accessed: 19.01.2020).

10. MeYou - a service for useful business acquaintances. https://www. meyou.ru (accessed: 19.01.2020).

11. Pittaway L., Robertson M., Munir K., Denyer D. and Neely A. Networking and innovation: a systematic review of the evidence. Interna- 
tional Journal of Management Reviews, 2004, no. 5-6: pp. 137-168. DOI:10.1111/j.1460-8545.2004.00101.x

12. Rainer A., Elgar F. Business Networking Systems: Characteristics and Lessons Learned. International Journal of Electronic Commerce, 2000, no. 5:2, pp. 7-27. DOI: 10.1080/10864415.2000.11044202

13. Schoonjans B., Van Cauwenberge P. \& Vander Bauwhede H. Formal business networking and SME growth. Small Bus Econ, 2013, no. 41, pp. 169-181. DOI:10.1007/s11187-011-9408-6

14. Wilson J. Social networking: the business case. Engineering \& Technology, 2009, no. 4, pp. 54-56. DOI: 10.1049/et.2009.1010

15. Worksup - Interaction and Networking App for Events: website https:// www.worksup.com (accessed: 19.01.2020).

\section{ДАННЫЕ ОБ АВТОРЕ}

Сухоруков Николай Николаевич, бакалавр кафедры «Информационные системы в экономике»

Волгоградский государственный технический университет Проспект им. В.И. Ленина, 28, г. Волгоград, 400005, Российская Федерация

nick-su26@mail.ru

\section{DATA ABOUT THE AUTHOR}

Sukhorukov Nikolay Nikolaevich, bachelor of the department «Informatics systems in economics»

Volgograd State Technical University

28, Lenin pr., Volgograd, 400005, Russian Federation

nick-su26@mail.ru

SPIN-code: 5395-2517

ORCID: 0000-0002-3835-9727 